\title{
Review of: "Effects of seed priming treatments on the germination and development of two rapeseed (Brassica napus L.) varieties under the co-influence of low temperature and drought"
}

प्र

Potential competing interests: The author(s) declared that no potential competing interests exist.

By studying the effects of low temperature and drought on the morphological indicators, physiological and biochemical and endogenous hormone content changes during the germination of rapeseeds, the effects of stress on rapeseed and the role of added hormones, $\mathrm{CaCl} 2$ and SNP in resisting drought are well revealed. I hope that the author can further study the effect of adding various hormones, $\mathrm{CaCl} 2$ and SNP on the drought-occurring rapeseed.

For this article, there are the following questions that need to be improved by the author:

1. The specific time of the germination test is $5 d$ or $7 d$ or $10 d$, etc.

2. The acquisition of photosynthetic stomata parameters and fluorescence parameters, and whether the germination test can meet these indicators

3. Obtain the test materials for endogenous hormone content, how to deal with it, and whether the germination box can meet these indicators should be specified.

4. Does the addition of $300 \mathrm{ng} / \mathrm{L} \mathrm{GA}$ and $89.4 \mathrm{~mol} / \mathrm{L} \mathrm{SNP}$ achieve relief from drought through changes in morphology, changes in physiological and biochemical indicators, and changes in endogenous hormones? It should be specific and clear, and how are the changes in morphology, Physiology and Biochemistry... In general, this article has solved the drought stress of rapeseed by starting with additives to promote the rape against drought. However, this method is relatively limited, and it is hoped that an effective and suitable additive formula can be found. 